\title{
CONGEDO MUSICALE
}

\author{
LUCIANO MARTINI (*)
}

\begin{abstract}
RIASSUNTO - L'ultima parte dell'incontro è dedicata alla presentazione di due DVD che permetteranno di ascoltare e vedere Arturo Toscanini mentre dirige la sua orchestra americana, la NBC Symphony, in due interessanti frammenti di composizioni di Giuseppe Verdi e Richard Wagner. Ascolteremo di Verdi l' "Inno delle Nazioni", una cantata scritta negli anni 1861-1862 su un testo di Arrigo Boito; di Wagner un brano dal frammento di "Morte di Sigfrido e Marcia funebre", tratto dal Götterdämmerung. Il testo sottolinea il ruolo del Maestro Toscanini nell' introdurre la musica di Wagner nel nostro paese, e nel presentare l'arte di Verdi, con un approccio critico e intellettuale che mancava nella "routine" generica del XIX secolo.
\end{abstract}

ABSTRACT - We end up our meeting presenting two DVD which will enable to listen and see Arturo Toscanini conducting his american orchestra, the NBC Symphony, in two interesting fragments of compositions of Giuseppe Verdi and Richard Wagner. Verdi will be represented by the "Hymn of the Nations", a cantata written in the years 1861-1862 on a text by Arrigo Boito; and Wagner by the fragment "Siegfried's Death and Funeral March" taken from the Götterdämmerung. The written text underlines the role of Maestro Toscanini in introducing Wagner's music in our country, and in showing the art of Verdi with a critical and intellectual approach which was missing in the generic "routine" of the nineteenth century.

L'ultima parte della nostra riunione è rappresentata da un "Congedo Musicale" dai due musicisti di cui abbiamo celebrato il bicentenario della nascita.

Ascolteremo alcune loro composizioni dirette da Arturo Toscanini, che, come vedremo, ha potuto avere più di un contatto per-

(*) Istituto Lombardo Accademia di Scienze e Lettere, Università degli Studi di Milano. 
sonale con Giuseppe Verdi ed è stato, in un secondo tempo, il primo interprete ad avvicinarsi alla sua musica con un criterio storico-critico che prese il nome, secondo noi non troppo appropriato, di "Verdi Renaissance".

Per quanto riguarda Wagner, il Maestro parmigiano è stato, all'inizio della sua carriera, in un'epoca in cui non tutti in Italia erano propensi ad accettare il verbo del compositore di Lipsia, uno dei suoi più ferventi e ammirati sostenitori. Egli diresse ripetutamente molti estratti sinfonici delle sue opere nei suoi innumeri concerti di quell'era ormai lontana, nonché molte prime esecuzioni integrali, nel nostro paese, dei suoi più significativi capolavori. Wagner rimarrà poi sempre presente, nella sua lunga e lusinghiera carriera, fino al termine della sua attività. Non dimentichiamo che l'ultimo suo concerto pubblico, quello tenuto a New York il 4 aprile del 1954, era tutto dedicato a musiche di Wagner. Sarà una combinazione, ma anche l'ultima volta che diresse alla Scala (19 settembre 1952), aveva scelto un programma tutto wagneriano: forse aveva il presentimento di non poter più ritornare, e desiderava quindi concludere così, con un forte riferimento a un suo idolo perenne, le sue purtroppo non numerose apparizioni sul podio del Piermarini, sul quale era ritornato, nel maggio 1946, per inaugurare la sala rinnovata del "suo" teatro, che era stata gravemente danneggiata dai bombardamenti aerei dell'agosto 1943.

Nel mio intervento toccherò alcuni punti salienti della vita musicale di Arturo Toscanini in quanto ritengo che, essendo passati più di 50 anni dalla sua scomparsa, sia doveroso ripulire la sua leggenda da molte fantasiose e non giustificate aggiunte. Dedicherò la mia analisi soprattutto ai primi anni della sua carriera perché sono quelli in cui si svolgeva la non silente battaglia culturale fra verdiani e wagneriani. Premetto che sono orgoglioso di sottolineare che l'audizione delle musiche dei due compositori protagonisti del nostro convegno avverrà nel ridotto della Scala che porta il nome del grande Maestro, e sotto la vigile sorveglianza della sua effigie magnificamente scolpita nel marmo imperituro di Adolfo Wildt.

Toscanini nasce a Parma nel 1867 e sale la prima volta su un podio direttoriale nel 1886 a Rio de Janeiro durante la "tournée" di una compagnia italiana guidata dall'impresario Claudio Bianchi che lo aveva ingaggiato come primo violoncello. Durante la stagione, il pubblico aveva più volte fischiato il direttore d'orchestra brasiliano Leopoldo Miguez che, per questo, si era dimesso dalle sue funzioni. Alla prima 
dell'Aida si presentò quindi sul podio un sostituto, un certo Carlo Superti, che però non ebbe migliore ventura del predecessore: tanto che fu obbligato ad abbandonare l'opera appena iniziata. Spinto dai suoi colleghi orchestrali, Toscanini salì sul podio e, per prima cosa, chiuse la partitura, sicuro di conoscerla tutta a memoria. Ovviamente aveva già in mente di non continuare la sua vita da semplice orchestrale, e stava tenacemente preparandosi alla nuova carriera, in un'epoca in cui la direzione d'orchestra non era materia di insegnamento nei Conservatori del Regno.

Rientrato in Italia abbandona il suo strumento e incomincia una vita di direttore-ospite trasferendosi in ogni città ove venisse invitato per la locale stagione lirica. Era molto richiesto, perché l'episodio brasiliano aveva avuto una grande risonanza nell'attentissimo mondo dei nostri teatri operistici ottocenteschi.

Tuttavia il 5 febbraio 1887 tornerà in orchestra, alla Scala, per la prima esecuzione dell'Otello di Verdi. Durante una delle prove, dirette da Franco Faccio, l'indivisibile sodale di Arrigo Boito, Verdi, che curava ogni particolare della preparazione del suo nuovo capolavoro, si avvicina all'orchestra - che allora era a livello della sala e non nel golfo mistico come la vediamo oggi - e, indirizzandosi a Toscanini, lo rimprovera per aver suonato troppo piano un passaggio del "solo" dei quattro violoncelli che prelude al grande duetto d'amore del primo atto "Già nella notte densa". Il giovane Arturo, che era, per sua natura, attentissimo alla lettura di tutte le indicazioni agogiche delle parti che aveva sul leggio, rispose: Maestro ma Lei, in questo punto, ha scritto “pianissimo". A Giuseppe Verdi rimase possibile una sola risposta: Sì ma è un pianissimo verdiano, volendo con questo indicare che, nella musica di Verdi, tutte le note debbono essere perfettamente udibili. L'episodio è certamente vero: oggi lo possiamo ascoltare in "internet" direttamente dalla voce di Arturo Toscanini che, nel 1946, durante una prova de La Traviata, lo racconta, agli orchestrali della NBC, la sua ultima orchestra americana, dopo essersi irritato con il comparto dei violini primi che, secondo lui, avevano emesso dei suoni troppo deboli e non adatti alla concezione verdiana.

Anche il secondo incontro fra i due grandi fu un incontro... didattico. Toscanini, che, pur non essendo presuntuoso, non era di certo umilissimo, aveva per Verdi una ammirazione e un rispetto analoghi a quelli che il buon Giuseppino rivolgeva ad Alessandro Manzoni, al cui nome, quando lo pronunciava, faceva precedere il forte aggettivo qua- 
lificativo "il santo". Il Maestro che, nel frattempo, era stato assunto come direttore stabile dal Teatro Regio di Torino (dove rimarrà dal 1895 al 1898) si accingeva, nel mese di maggio del 1898, a presentare a quel pubblico l'ultima composizione di Verdi: i quattro Pezzi Sacri. Era il suo primo approccio a quella straordinaria partitura - le ultime note scritte da Verdi sul pentagramma! - e voleva alcune delucidazioni sui tempi e sulle sonorità che il compositore desiderava per la più complessa delle quattro composizioni, il Te Deum. Per questo chiese un appuntamento e si recò, accompagnato dal critico musicale de "La Stampa" Giuseppe Depanis, a Genova dove, a Palazzo Doria, Verdi possedeva un magnifico appartamento (la sala da pranzo genovese venne da Verdi stesso devoluta alla Casa di Riposo per Musicisti di Milano, dove è tuttora visibile). Sperò invano che il compositore si sedesse al pianoforte. Toccò invece a lui eseguire tutto il Te Deum e lo fece con l'elasticità dinamica e con le sonorità ferventi che il sentimento gli suggeriva. Alla fine Verdi disse: Bravo, così lo ho pensato. Toscanini, riferendosi specificamente a un rallentando che gli sembrava essenziale nell'agogica del pezzo, domandò al compositore perché non lo avesse scritto come indicazione per gli esecutori. Per timore che venisse eseguito troppo lento, fu la risposta secca di Verdi. Toscanini ebbe così il via libera per la propria interpretazione e il Te Deum divenne uno dei suoi pezzi preferiti. Nei concerti che terrà alla Scala dal 1946 al 1952 lo eseguirà ben due volte: nel concerto inaugurale e in quello del 26 giugno 1950, in cui diresse, per celebrare l'Anno Santo, anche la Messa da Requiem. ${ }^{1}$ La testimonianza verbale di Verdi, pronunciata, fra l'altro, in presenza di un importante testimone, è stata trasferita, di pugno del Maestro, sulla partitura da lui usata ogni volta che tornava a dirigere quella composizione. Il bel volume rilegato, che ho potuto stu-

1 La stagione dei Concerti di Primavera della Scala del 1950 è rimasta memorabile nella storia del Teatro. Abbiamo potuto ascoltare, oltre al concerto verdiano di Toscanini: 1) La Passione Secondo San Matteo di Johannes Sebastian Bach diretta da Issay Dobrowen; 2) Il Magnificat di Claudio Monteverdi e il Requiem di Wolfgang Amadeus Mozart diretti da Guido Cantelli; 3) La Missa Solemnis di Ludwig van Beethoven eseguita dall'Orchestra e dal Coro del Musikverein di Vienna diretti da Herbert von Karajan; 4) La Messa in si minore di Johannes Sebastian Bach con gli stessi complessi viennesi guidati da Herbert von Karajan: in quella serata fu presente il grande contralto inglese Kathleen Ferrier (1912-1953), nella sua unica apparizione alla Scala; 5) Il Requiem Tedesco di Johannes Brahms diretto da Victor de Sabata. 
diare attentamente, è stato preso in consegna, assieme a tutti i documenti e gli spartiti toscaniniani che erano rimasti nella villa di Riverdale, ultima dimora del Maestro, dalla "New York Public Library for the Performing Arts" sita al Lincoln Center di New York, proprio accanto al nuovo "Metropolitan Theatre". Non è difficile per gli studiosi essere ammessi ad analizzare i cospicui documenti di questo Legato Toscanini.

Il prolungato soggiorno al Teatro Regio di Torino nel triennio 1895 1898 fu molto importante per il Maestro. Infatti, il contratto che aveva firmato con l'impresario Piontelli lo obbligava ad alternare la direzione di opere complete a concerti sinfonici da condividere, a turno, con altri direttori del calibro di Giuseppe Martucci (1856-1912) e di Charles Lamoureux (1834-1899), per citare solo due nomi di impegno. Questo lo portò a estendere il proprio repertorio aggiungendovi composizioni e opere di autori illustri dei secoli precedenti, di specializzarsi nei classici sette-ottocenteschi e di non trascurare i compositori contemporanei. Nella capitale sabauda, poi, egli conobbe molti musicisti della nuova generazione. Si legò di particolare amicizia con Alfredo Catalani (18541893) e con Leone Sinigaglia (1868-1944): di entrambi si ricorderà fino al termine della propria carriera con ottime incisioni effettuate anche nell'ultimo periodo americano. Conobbe anche Giacomo Puccini (18581924) che gli affidò, nel 1896, la prima esecuzione assoluta della sua Bobème. Fu a Torino che diresse, per la prima volta nel nostro paese, il Crepuscolo degli Dei di Wagner (22 dicembre 1895), che, eseguito in lingua italiana, ebbe ben 21 repliche. Da notare che già la prima sera venne richiesto il "bis" della Marcia Funebre di Sigfrido, che Toscanini non concesse, secondo quella che diverrà, più avanti, una sua regola assoluta. Non si trattava comunque della prima opera wagneriana completa che Toscanini mettesse in repertorio. Il Crepuscolo torinese era stato infatti preceduto, sempre nel 1895, dal Tannbäuser che aveva diretto al Teatro Carlo Felice di Genova. L'anno successivo aggiunse il Tristano e Isotta, affiancato poi, nella stagione seguente, da La Valchiria. Per la fama di colto e attento musicista che ormai lo circondava, e per il vasto repertorio che aveva incastonato nella propria memoria, gli vennero affidati ben 43 concerti sinfonici, da dirigere al ritmo di due alla settimana, per accompagnare il successo della Grande Esposizione di Torino del 1898.

A questo punto divenne inevitabile il passaggio alla Scala di Milano, dove fu invitato a prendersi cura dell'orchestra dal grande imprenditore Giulio Gatti Casazza (1869-1940), che agiva in nome dei proprietari del teatro, i così detti "palchettisti", guidati allora dalla 
migliore aristocrazia della città. Particolarmente attenti alle fortune del teatro furono, in quell'epoca, per due generazioni consecutive, i conti Guido (1838-1902) e Uberto (1871-1923) Visconti di Modrone. Limiteremo la nostra analisi al primo soggiorno scaligero del Maestro che, per rispettare gli impegni torinesi, avrà un prologo molto significativo nel 1896 e diventerà esclusivo dal 1898 al 1903. Dato il suo legame contrattuale, le sue prime apparizioni come direttore wagneriano non avverranno con l'esecuzione integrale di un'opera completa, ma invece inserendo, in vari programmi concertistici, spezzoni dei loro contenuti lirico-sinfonici. Con questo il Maestro pensava di offrire al pubblico scaligero, tradizionalmente legato al melodramma italiano e al "Grand Opéra" francese, una specie di graduale rodaggio nei confronti della imponente drammaturgia wagneriana. L'inizio di questo processo avverrà il 26 aprile del 1896 con l'esecuzione del Prologo del Crepuscolo degli Dei, esecuzione alla quale parteciperanno diversi interpreti di canto, fra cui il tenore Giuseppe Borgatti che diverrà, da quel momento, il tenore wagneriano preferito da Toscanini. ${ }^{2}$ Il lungo fram-

2 La prima scrittura che Giuseppe Borgatti (1871-1950) ricevette dalla Scala fu per la prima rappresentazione assoluta dell'opera Andrea Chénier (28 marzo 1896) di Umberto Giordano (1867-1948). Un mese dopo venne prescelto da Toscanini per le tre esecuzioni del Prologo del Crepuscolo degli Dei che avrebbe presentato alla Scala in forma di concerto (26 aprile, 3 e 10 maggio 1896). Nacque così, con la parte di Sigfrido cantata da Borgatti, il primo "Heldentenor" italiano. Come vedremo Toscanini lo vorrà anche come Lohengrin e come Tristano quando eseguirà alla Scala le due opere wagneriane in forma scenica. Borgatti ebbe l'onore di essere il primo tenore italiano a essere invitato a cantare, nel 1904, nel tempio wagneriano di Bayreuth. Qualche anno più tardi Borgatti cantava ancora sotto l'egida di Toscanini. Una sera il Maestro si accorse che il suo tenore gli sfuggiva, non seguiva il suo gesto ed era in ritardo a ogni entrata: cose, ovviamente, che solo il terribile orecchio musicale del Maestro riusciva ad avvertire e che il grande pubblico non percepiva. Nell'intervallo Borgatti gli confessò che, quella sera, aveva un forte dolore a un occhio e che gli sembrava di vedere delle ombre. Il Maestro, che conosceva i danni che potevano derivare da una diminuzione della vista (la sua miopia gravissima era la ragione del suo dirigere a memoria senza lo spartito davanti), gli fece coraggio e lo incitò a continuare la recita. Fece anche cambiare quella che oggi chiamiamo la regia, per permettere a Borgatti di cantare più vicino al proscenio e di avere quindi meno problemi nel seguire i suoi gesti. Era, per il grande tenore, il primo attacco del glaucoma che, di lì a qualche anno (nel 1915), lo obbligherà ad abbandonare le scene. Sopravvisse cieco fino al 1950 facendo, fino quasi alla fine dei suoi giorni, il maestro di canto. 
mento wagneriano era stato preceduto da una Sinfonia di Haydn e dallo Schiaccianoci di Ciaikovsky. Poiché "repetita juvant", il Prologo del Crepuscolo venne ripreso il 3 e il 10 maggio dello stesso anno in due contesti musicali diversi. La prima volta con la $1^{a}$ Sinfonia di Beethoven, la Ouverture Tragica di Brahms e la Suite Holberg di Grieg; la seconda con la 6 a Sinfonia di Schubert, due Intermezzi tratti dall'opera Cleopatra di Luigi Mancinelli, nonché la Danza Macabra di Saint-Saëns. ${ }^{3}$

Il quarto e ultimo concerto di questa prima serie toscaniniana nel teatro milanese presenterà, invece, di Wagner, il Preludio del Parsifal e Il Mormorio della Foresta tratto dal Sigfrido. Ritenendo che questa prima imbandigione del sinfonismo wagneriano avesse iniziato per lo meno a scalfire, se non a rompere, il ghiaccio con il pubblico milanese, il Maestro decise di passare, il 26 dicembre 1898, alla presentazione di un'opera completa. Scelse I Maestri Cantori di Norimberga, un capolavoro che non aveva ancora diretto e che poi prediligerà fino alla fine della propria carriera (negli anni '30, dirigerà quest'opera anche al Teatro wagneriano di Bayreuth e al Festival di Salisburgo). Da notare che Die Meistersinger sarà in assoluto la prima opera completa che Toscanini dirigerà alla Scala. Per introdurre nel mondo dello spettacolo un concetto al quale ci siamo abituati in tempi recenti, quello della "par condicio", dirigerà però subito dopo (11 marzo 1899) il suo primo Falstaff scaligero.

Il primo concerto di Toscanini nella stagione del 1899 verrà programmato per il 16 aprile e sarà dedicato ai Pezzi Sacri di Verdi. Nel periodo di cui ci stiamo occupando si verificheranno due altri suoi memorabili interventi verdiani: il concerto del 27 gennaio del 1902 quando, per celebrare il primo anniversario della scomparsa del compositore, diresse, molto probabilmente per la prima volta, il suo Requiem; e poi l'esecuzione pubblica all'aperto, nel piazzale davanti al Famedio del Cimitero Monumentale di Milano, nella quale diresse, il 26 febbraio 1902, con l'orchestra e il coro della Scala, il mirabile canto

3 È interessante sottolineare che molti dei compositori rappresentati in questi concerti erano ancora viventi, a partire da Johannes Brams (1833-1897) che morirà l'anno seguente a quello in cui apparve nel programma toscaniniano. Il più vecchio fra gli altri, Camille Saint-Saëns (1835-1921), sarà attivo ancora per più di un ventennio; così come Luigi Mancinelli (1848-1921). Il più prossimo alla morte era Edvard Grieg (18431907), che potrà comporre solo per un altro decennio. 
del Nabucco "Va pensiero sull'Ali Dorate" in occasione della traslazione delle salme di Giuseppe Verdi e di sua moglie Giuseppina Strepponi nella Cripta della Casa di Riposo per Musicisti creata dalla generosità del grande bussetano. Il versatile artista Lodovico Pogliaghi, che era pittore e scultore molto ricercato, aveva appena terminato i lavori per renderla adatta a onorare per sempre il riposo del nostro grande genio. ${ }^{4}$

Il 6 maggio 1899 Toscanini riprenderà la presentazione in concerto di pezzi scelti delle opere di Wagner, dirigendo l'Ouverture del Tannäuser che sarà seguita, pochi giorni dopo (11 maggio 1899), dal Duetto del secondo atto del Tristano e Isotta definito, nella locandina dell'epoca, "Scena d'amore". L'anno successivo, il 22 aprile 1900, appariranno in programma Il Giardino di Klingsor tratto dal Parsifal e l'Entrata degli dei nel Walhalla, scena finale dell'Oro del Reno. Il 27 aprile 1900 ritornerà al Götterdämmerung dirigendo la Morte e Marcia Funebre di Sigfrido e chiuderà il suo concerto con l'Ouverture dei Meistersinger. Il 12 maggio aggiungerà ai programmi scaligeri la prima esecuzione della Faust Ouverture e quella della Cavalcata delle Valchirie, perno musicale di tutta l'opera omonima. Il 14 maggio riprenderà, infine, La Marcia Funebre di Sigfrido e l'Entrata degli dei nel Walhalla.

Parallelamente alle presentazioni in concerto inizierà ad affermarsi anche la produzione di opere complete. La stagione operistica del 1900 (che, come quelle che l'avevano preceduta, veniva definita "di Carnevale" e iniziava quindi il giorno di Santo Stefano dell'anno precedente) $)^{5}$ si aprirà con il Sigfrido (naturalmente il tenore sarà Borgatti) e

4 Lodovico Pogliaghi (1857-1950) fu un artista colto e poliedrico che sapeva alternare, e abbinare fra loro, la scultura e la pittura. Fra le sue opere più prestigiose si ricordano: la porta centrale del Duomo di Milano; la Pietà nella Cappella Espiatoria che a Monza ricorda l'assassinio di Umberto $1^{\circ}$ di Savoia; il Gruppo della Concordia per l'Altare della Patria a Roma (noto anche come il Vittoriano); le porte bronzee di Santa Maria Maggiore a Roma, suo ultimo lavoro. Preparò inoltre i cartoni per diverse opere musive presenti in alcune Cappelle del Cimitero Monumentale di Milano e per i mosaici della Cripta della Casa di Riposo per Musicisti - Fondazione Verdi. Per la sua duttilità e per la sua preparazione storica, fu anche apprezzato scenografo. Alla Scala ideò e dipinse le scene per la prima rappresentazione del Nerone di Arrigo Boito (1924), che Toscanini fece poi riutilizzare nella serata con cui, alla Scala, volle ricordare, nel 1948, il trentennale della morte del compositore.

5 Dalla sua inaugurazione nel 1779 fino al 1902, infatti, le stagioni operistiche 
proseguirà il 27 gennaio 1900 con il Lohengrin. ${ }^{6}$ Fra le due opere wagneriane, sempre per "par condicio", il Maestro aveva provveduto a infilare una bella ripresa dell'Otello (27 dicembre 1899).

La stagione successiva, quella del 1901, vedrà la prima produzione alla Scala del Tristano e Isotta (29 dicembre 1900, sempre con Borgatti protagonista) e sarà caratterizzata dall'assenza di opere di

della Scala iniziavano il 26 dicembre. Rare furono le eccezioni. Negli anni successivi, la data dell'inaugurazione delle singole stagioni divenne variabile, per il fatto che gli Impresari tendevano a iniziare le stagioni più precocemente per aumentare il numero degli spettacoli, e quindi gli incassi, visto che avevano una data di chiusura della stagione che, all'epoca, non era facilmente travalicabile: la fine del Carnevale Ambrosiano. All'epoca in cui il Teatro era una proprietà privata e apparteneva ai "palchettisti", questi sceglievano infatti un Impresario che poteva cambiare di anno in anno o essere rinominato per diverse stagioni consecutive. L'impresario doveva occuparsi di scegliere le opere da rappresentare, di creare un'orchestra e un coro, di scritturare i cantanti più graditi al pubblico, senza dimenticare che le Opere erano di solito seguite da un Balletto: nella selezione delle ballerine intervenivano come interessati consulenti - e sempre con cospicua tenacia - i più accesi palchettisti. La durata della stagione dipendeva da tutte queste variabili e, ovviamente, anche dagli incassi effettuati. Da quando, nel 1921, la Scala è diventata un Ente Autonomo del Comune di Milano - grazie all'opera di Uberto Visconti di Modrone, di Arturo Toscanini e del futuro Segretario Generale, l'ingegnere Angelo Scandiani (1872-1930) (che aveva appena terminato una non modesta carriera di baritono ricercato da teatri anche di rilievo) - prevalse il criterio di effettuare stagioni più prolungate al fine di dare un lavoro continuativo alle maestranze, che comunque erano pagate in base a contratti annuali. Per questo, l'inizio della stagione veniva anticipato a diverse date non festive di novembre o di dicembre. Tuttavia il Falstaff con cui Toscanini inaugurò la nuova epoca e la nuova struttura organizzativa venne eseguito il 26 dicembre del 1921, giorno di Santo Stefano. La data di inizio alla sera di Sant'Ambrogio ( 7 dicembre) venne scelta per la prima volta per le stagioni del 1930 (7 dicembre 1929) e 1931 (7 dicembre 1930). Della seconda scelta è stata sicuramente responsabile la Signora Anita Colombo, da sempre stretta collaboratrice di Toscanini, che era stata nominata Segretario Generale del teatro dopo la morte, nel 1930, dell'ingegnere Scandiani. Il suo regno fu comunque breve, durò solo un anno, perché il governo fascista creò, alla fine del 1930 la nuova figura del Sovrintendente. Dalla stagione del 1932 fino alla Seconda Guerra Mondiale l'inaugurazione avvenne sempre il 26 dicembre. Dopo la guerra e la ricostruzione del Teatro le stagioni sono iniziate a Santo Stefano dal 1947 al 1950. La riscoperta del 7 dicembre - Sant'Ambrogio scaligero avvenne nel 1951 e perdura tuttora.

6 Non era la prima scaligera: dopo il noto e discusso esordio bolognese del 1 novembre 1871, Franco Faccio aveva presentato alla Scala il Cavaliere del Cigno nel 1873 e poi ancora nel 1889 . 
Verdi. Si potranno ascoltare invece, dirette da Toscanini, il Mefistofele di Arrigo Boito, la Bohème di Giacomo Puccini e Le Maschere di Pietro Mascagni. ${ }^{7}$ Anche la stagione del 1902 sarà inaugurata da Wagner (26 dicembre 1901) con la prima edizione integrale de La Valchiria. Questa volta il pareggio verrà ottenuto con il Trovatore che andrà in scena il 9 febbraio 1902. Infine, Toscanini chiuderà la sua ultima stagione di quell'epoca, quella del 1903, con il Parsifal (12 aprile1903), dopo aver eseguito di Verdi la Luisa Miller e il Ballo in Maschera.

A questo punto Toscanini ha memorizzato e diretto, nei primi 36 anni della sua vita, ben 9 opere complete di Richard Wagner!

Le successive tappe della vita di Toscanini si possono così riassumere:

- 1905-1907: Secondo periodo di Direzione dei Concerti della Scala.

- 1908-1915: Direttore Artistico del "Metropolitan Theatre" di

New York, dove era diventato Direttore Generale Giulio Gatti Casazza.

- 1921-1929: Direttore Artistico della Scala dove era stato nominato Segretario Generale l'ingegnere Angelo Scandiani.

- 1929-1936: Direttore Artistico della "New York Philarmonic Orchestra”.

- 1930-1931: Primo musicista non tedesco a essere invitato a dirigere al Festival Wagneriano di Bayreuth. ${ }^{8}$

7 Si trattò chiaramente di una apertura alle nuove generazioni e all'opera postverdiana. Il Mefistofele di Arrigo Boito (1842-1918) aveva avuto la sua prima esecuzione alla Scala nel 1867; la Bohème di Giacomo Puccini apparve nel 1896, subito dopo il trionfo torinese della prima assoluta dell'opera diretta da Arturo Toscanini. Le Maschere di Pietro Mascagni (1863-1945) erano invece una novità assoluta la cui prima, per un capriccio dell'autore che, dopo l'immenso successo ottenuto nel 1890 dalla sua Cavalleria Rusticana al Teatro Costanzi di Roma, si considerava il nuovo re del mondo della lirica, avveniva simultaneamente in sei teatri: alla Scala (che prevalse in quanto, oltre alla direzione di Toscanini, aveva Enrico Caruso come protagonista), al Carlo Felice di Genova, al Regio di Torino, al Costanzi di Roma, alla Fenice di Venezia e al Filarmonico di Verona. Al San Carlo di Napoli fu rappresentata con due giorni di ritardo per ragioni organizzative.

8 Al Teatro wagneriano di Bayreuth venne invitato la prima volta per i Festspiele del 1930, in cui diresse Tannbäuser e Tristan und Isolde. Venne invitato anche per i Festspiele del 1931 e vi diresse il Tannbäuser e il Parsifal. Non accettò l'invito per 
- 1935-1936-1937: Primo Direttore ospite straniero del Festival di Salisburgo.9

- 1937-1954: Direttore Artistico dell'Orchestra della NBC ("National Broadcasting Corporation"), creata per lui da David Sarnoff, fondatore e maggiore azionista di quella rete radiofonica. - 1957: morirà a New York, nella sua bella Villa di Riverdale nei Bronx.

Concludiamo con delle brevi note su l'Inno delle Nazioni, una cantata profana di Giuseppe Verdi, che non viene frequentemente eseguita, ma che vi permetterà di apprezzare la maestria direttoriale di Arturo Toscanini. Si tratta di una composizione storicamente importante perché segna la prima collaborazione di Verdi con Arrigo Boito, che sarà poi il librettista delle sue due ultime opere, Otello e Falstaff. L'origine della composizione fu l'offerta che il maestro ricevette nel

il 1932 perché era evidente che il Teatro era ormai dominato dai nazisti. I direttori d'orchestra italiani che furono invitati in seguito sono stati: Victor de Sabata nel 1939; Alberto Erede nel 1968; Giuseppe Sinopoli nel 1985, nel 1998 e nel 2000; Daniele Gatti nel 2008, nel 2009 e nel 2010.

9 Al Festival di Salisburgo Toscanini venne invitato, per la prima volta, nel 1935 e vi diresse il Falstaff di Verdi e il Fidelio di Beethoven. Il personaggio di Falstaff venne affidato a Mariano Stabile (1888-1968), al quale aveva insegnato personalmente la parte per l'inaugurazione (26 dicembre 1921) del suo periodo d'oro alla Scala. Il Fidelio ebbe come interprete principale la famosissima Lotte Lehamann (1888-1976). Toscanini tornò a Salisburgo nel 1936 ed eseguì ancora Falstaff e Fidelio, aggiungendo però anche Die Meistersinger. La sua ultima stagione salisburghese fu quella del 1937 in cui ripropose le tre opere dell'anno precedente alle quali fu richiesto di aggiungere Die Zauberflöte. In poche parole: avevano affidato a lui, in quello che era all'epoca il Festival più importante del mondo, e al quale erano presenti almeno due altri massimi direttori di lingua e stile viennese, Bruno Walter (1876-1962) e Fritz Busch (18901951), le opere chiave del repertorio tedesco. Bruno Walter, che era diventato grande amico del Maestro, oltre che suo sincero ammiratore, cercò in tutti i modi di invitarlo ancora per il Festival del 1938. Ma Toscanini fu irreducibile e già nel novembre del 1937 aveva rifiutato, sia pure con motivata gentilezza. Vivendo a New York conosceva la situazione politica europea meglio di chi viveva a Vienna. Infatti, prima dell'inizio del Festival 1938, Hitler invaderà l'Austria proclamandone l'annessione ("Anschluss”) alla Germania (12 marzo 1938). I due direttori si ritroveranno, nel 1938, al nuovo Festival di Lucerna, voluto da Toscanini e dai membri del Quartetto di Adolf Busch (18911952) per dare una occupazione ai musicisti ebrei scacciati dai loro posti di lavori dall'egemonia della Germania Nazista. I componenti del famoso quartetto Busch divennero le prime parti della nuova improvvisata orchestra. 
1861 dal Comitato Organizzatore affinché scrivesse un pezzo celebrativo per la "International Exhibition" che si progettava di tenere a Londra nel 1862. Nel febbraio del 1861, mentre era a Parigi, Verdi venne avvicinato da Arrigo Boito, allora giovane ventenne e poeta scapigliato: aveva con sé una lettera di presentazione e raccomandazione della storica amica milanese del compositore, Clarina Maffei. ${ }^{10}$ Evidentemente, in uno dei loro incontri, i due parlarono dell'argomento e Verdi, che aveva meditato a fondo la richiesta inglese e si era già fatto un'idea di cosa avrebbe potuto creare di originale per una manifestazione internazionale di tale importanza, decise di invitare il poeta a presentargli un testo per una composizione che suggerisse il trionfo della pace sulla guerra. Boito preparò un breve poemetto per un cantante solista, accompagnato da un coro misto e da una robusta orchestra. Alla musica, ovviamente, pensò Verdi che scrisse, per il tenore solista, una bellissima perorazione seguita da un coro forte come una preghiera: "Signor che su la terra rugiade spargi e fior". Infine, per dare un tono internazionale alla sua composizione, Verdi volle concluderla con un coro in cui introdusse un motivo fugato in cui vengono citate le note iniziali dell'Inno Inglese "God save the King" (o, se preferiamo, God save the Queen, visto che sul trono, in quel momento, sedeva la longeva Regina Vittoria), subito seguite da

10 Elena Clara Antonia Carrara Spinelli (1814-1886) è oggi più nota come Clara o Clarina Maffei, dal nome del marito Cavalier Andrea Maffei (1798-1885). È rimasta celebre per aver fondato, a Milano, il più importante salotto letterario dell'ottocento, che ebbe sede dapprima in via Dei Tre Monasteri e finalmente in via Bigli, nel bel palazzo all'angolo con via Manzoni ben riconoscibile per la sua monumentale "porte cochère”. In estate, gli incontri avvenivano a Clusone nella villa Carrara Spinelli che Clarina aveva ereditato dai propri genitori. Il salotto di casa Maffei svolse un grande ruolo nella preparazione e divulgazione del nostro Risorgimento, prima e dopo le 5 Giornate. Era frequentato assiduamente da Tommaso Grossi (1791-1853), da Massimo D'Azeglio (1798-1886) e da Francesco Hayez (1791-1882), che ci lasciò un ritratto della contessa. Molto influente nel salotto era anche Carlo Tenca (1816-1883) giornalista e pensatore di spicco: fra lui e Clarina sorse una romantica passione. Compariva saltuariamente anche Alessandro Manzoni (1785-1873) di cui Clarina frequentava abitualmente la casa; fu lei ad accompagnarvi Verdi al primo incontro con il suo "santo", come soleva definire l'autore dei Promessi Sposi. Andrea Maffei, letterato e traduttore di drammi e di poemi, scrisse per Verdi il libretto de I Masnadieri, derivato da Die Raüber di Friedrich Schiller (1759-1805) e rimaneggiò, per renderlo più sakespeariano, il libretto del Macbeth di Francesco Maria Piave. 
quelle della Marsigliese, ${ }^{11}$ e, appena dopo, da quelle dell'Inno di Mameli, che tutti oggi conosciamo come Inno Nazionale della nostra Repubblica. Verdi vedeva sempre lontano: prevedeva che l'Italia unificata, nata nel 1861, dopo il movimento risorgimentale al quale aveva partecipato sia direttamente sia con la creazione di opere a sfondo patriottico, avrebbe prima o poi utilizzato quelle note sacre agli eroi della Repubblica Romana del 1849.

Toscanini diresse l'Inno delle Nazioni per la prima volta il 26 luglio 1915 in un memorabile concerto patriottico del coro e dell'orchestra della Scala all'Arena di Milano. Facevano parte del programma, oltre all'Inno, solo musiche verdiane del periodo risorgimentale. Lo scopo del concerto era quello di far sentire la presenza viva del popolo italiano ai nostri soldati che avevano appena attraversato il Piave per unirsi agli alleati della Triplice Alleanza nella Prima Guerra Mondiale.

L'edizione che vedremo e ascolteremo è quella trasmessa il 31 gennaio 1944, in uno dei concerti con la sua ultima orchestra americana, quella della NBC (Natinal Broadcasting Corporation), rinforzata, per l'occasione dal Westminster Choir, e dal tenore Jan Peerce. L'esecuzione fu anche filmata per essere incorporata in un cortometraggio che intendeva ringraziare gli italiani che si erano rifugiati negli Stati Uniti per sfuggire alle angherie nazifasciste di quel periodo. Nel film, che venne proiettato in Italia subito dopo il 25 aprile 1945 (lo vidi al cinema Meravigli nella via omonima ora scomparso), aveva una parte preponderante Randolfo Pacciardi già eroe della guerra di Spagna e poi personaggio-simbolo, da noi, del Partito Repubblicano. ${ }^{12}$ Per l'occasione, Toscanini ritenne opportuno aggiungere, dopo il fugato al quale abbiamo fatto cenno, l'Internazionale Socialista (che all'epoca era l'in-

11 Verdi sapeva benissimo che non avrebbe usato l'Inno nazionale francese operativo in quel momento. Sentiva però, da uomo libero, che quel canto era l'unico che sottolineasse il vero significato della Francia nel mondo: quello immortale, nato dalla rivoluzione francese del 1789. La Marsigliese, che era stato l'inno nazionale francese dal 1830 al 1856, venne sostituita con un canto che nessuno più ricorda da Napoleone III autonominatosi Imperatore dei francesi. Venne ripristinata, come Inno nazionale, solo nel 1876, dopo che la sconfitta di Sedan e la rivoluzione del 1870 avevano permesso di cacciare Napoleone "il piccolo".

12 Randolfo Pacciardi (1899-1991) fu un attivo antifascista e un uomo politico italiano. Si iscrisse all'allora piccolo Partito Repubblicano Italiano (PRI) nel 1915 quando aveva solo 16 anni. Personaggio sicuramente coraggioso fu volontario nei Bersaglieri 
no della Unione Sovietica e lo "Star-Bangled Banner", Inno Nazionale degli Stati Uniti, alleati dell'Inghilterra e della Francia nella seconda guerra mondiale. Questa parte non verdiana è stata eliminata successivamente.

Non mi risulta che Toscanini abbia diretto l'Inno delle Nazioni in altre occasioni oltre alle due che abbiamo ricordato.

La morte e la Marcia funebre di Sigfrido sono invece prese dal primo (guarda caso: un tutto-Wagner!) dei cinque concerti che Toscanini diresse a New York fra il 1948 e il 1952 per la televisione della National Broadcasting Corporation, proprietaria dell'orchestra che era stata creata per lui nel 1937. Questo, del 20 marzo 1948, fu in assoluto il primo concerto di un'orchestra sinfonica a essere trasmesso al mondo da una televisione. Altro primato di Toscanini! Nel presentare la nuova ambiziosa avventura, prima dell'inizio del concerto, David Sarnoff, fondatore e maggiore azionista della NBC, fece un breve discorso durante il quale, con commossa simpatia, disse: Siamo fortunati di aver con noi, in questa avventura, il maestro Toscanini che è ancora "a joung fellow" (un giovanotto). Il Maestro aveva 84 anni!

Fra le due riproduzioni che vedrete erano passati sette anni. Sono certo che non ve ne accorgerete!

nella Prima Guerra Mondiale e fu insignito di due medaglie d'argento e di una di bronzo. Antifascista convinto fin dal 1922, venne condannato a 5 anni di confino, ma riuscì a evadere ed espatriare. Rifugiato in Francia, nel 1936, fondò la Lega Antifascista per raccogliere volontari che volessero arruolarsi per combattere il Generale Franco durante la Guerra Civile Spagnola. Partecipò direttamente, a lato dei repubblicani, a quel conflitto, dove ebbe anche posizioni di comando. Fu ferito, ma, guarito, ritornò al suo battaglione. Dal 1941 al 1944 fu negli Stati Uniti, ove strinse una forte amicizia con Toscanini. Rientrato in Italia venne eletto Segretario del Partito Repubblicano Italiano (PRI). Fece parte dell'Assemblea Costituente e fu Ministro della Difesa dal 1948 al 1953. L'ho visto in almeno due cerimonie tenute a Parma quando, in Oltretorrente, venne inaugurata e poi trasformata in Museo la casa natale del Maestro. 